\title{
Emotional Recognition of EEG Signals based on Fractal Dimension
}

\author{
Xin $\mathrm{Xu}^{\mathrm{a}, \mathrm{b}, *}$, Meng $\mathrm{Cao}^{\mathrm{c}}$, Jiawei Ding ${ }^{\mathrm{c}}$, Hong $\mathrm{Gu}^{\mathrm{b}}$, and Wenjuan $\mathrm{Lu}^{\mathrm{c}}$ \\ ${ }^{a}$ School of Geographical and Biologic Information, Nanjing University of Posts and Telecommunications, Nanjing, 210023, China \\ ${ }^{b}$ School of Electronic and Optical Engineering, Nanjing University of Science and Technology, Nanjing, 210094, China \\ ${ }^{c}$ College of Telecommunications and Information Engineering, Nanjing University of Posts and Telecommunications, Nanjing, 210003, China
}

\begin{abstract}
A method based on fractal dimension is proposed to identify EEG emotional signals, and fractal dimension is introduced as an eigenvalue into emotion recognition research. The design experiment obtains the EEG raw data of the experimenter and uses the experimental video to locate and capture the effective signal from the original data. After the pre-electron interference and low-pass filtering are applied, the effective signal is subjected to principal component analysis. The dimension reduction dimension is obtained by reducing the dimension. The support vector machine (SVM) and K nearest neighbor (KNN) classification algorithm are used to classify the eigenvalues to obtain their respective accuracy. The results show that the EEG emotion recognition method based on fractal dimension can distinguish different emotions, and the highest accuracy rate is $83.33 \%$. Therefore, fractal dimension is feasible as the characteristic value of emotion recognition.
\end{abstract}

Keywords: electroencephalography; emotion recognition; principal component analysis; support vector machines; $K$ nearest neighbor

(Submitted on October 5, 2019; Revised on November 6, 2019; Accepted on November 15, 2019)

(c) 2019 Totem Publisher, Inc. All rights reserved.

\section{Introduction}

Expressing emotions is a daily activity that plays a critical role in learning processes, but it is difficult to give a precise definition of emotions. The importance of emotions is not only reflected in emotional intelligence, but also in human relationships (human-human interaction, HHI). Machines should be able to identify, understand, and recognize human emotional needs in the interactive phase for more efficient and pragmatic human-computer interaction. Some evidence [1] has suggested that, if the HMI can be as effective as the HHI, machines must have the ability to perceive human emotions. A patient's emotional state has a profound impact on the final outcome of medical care [2], because the patients' emotional state is an important factor in their mental and physical health. At present, some researchers have added an emotional part to the HMI in a human-machine interface that can receive data about facial expressions, sounds, and other relevant physiological indices (e.g., skin resistance and heart rate). There have been many situations in the process of interface implementation. For example, in the emotional recognition system based on GSR, the effect of sweating on the recognition result is greater than the effect of the emotion itself on the system, because sweating can greatly affect emotional recognition [3]. Currently, there are not many systems that use EEGs to identify emotional states, but the proposed EEG method can compensate for the shortcomings of emotional recognition mentioned above. Meanwhile, it has been widely recognized that this problem is based on wavelet analysis [4] and statistics methods [5] and can be solved by combining classification algorithms such as SVM and KNN.

This paper describes an emotional recognition based on fractal dimension and obtains the characteristic information of the EEG signal under different emotional states. Finally, the classification accuracy of different emotions is available under two categories. According to the system theory, the EEG time series can be regarded as originating from a system. In fact, there may be multiple independent subsystems involved in the formation of EEG in the brain, so it may be assumed that there are several independent systems. Since the brain is a volume conductor, the EEG measured at the scalp is the spatiotemporal superposition of the electrical activity of the $n$ systems. Dimension analysis extracts the information of the n-

* Corresponding author.

E-mail address: xuxin@njupt.edu.cn 
dimensional state space from the one-dimensional EEG time series obtained from the experiment. The data used in this paper are collected from 12 volunteers aged 20 to 24 . Volunteers are stimulated by watching videos in order to evoke different emotional reactions, which are recorded by special equipment. The second section describes the experiment and relevant information, the third section provides the data analysis, and the fourth section presents the conclusions.

\section{Emotional Model and Experimental Design}

\subsection{Emotional Model}

The process of emotion recognition includes information gathering, emotion modeling, and emotional feedback. A reasonable and effective sentiment model is the basic guarantee for comprehensive evaluation of emotional states. An emotional model is the basis and key for emotional analysis.

At present, in the field of emotional processing, the main models used are discrete emotion models, continuous emotion models, and cognitive-based emotion models.

1) Discrete sentiment model: A discrete sentiment model is constructed based on the idea of classification modeling. The model believes that there are several basic emotions in the human brain. These emotions are generally easy to be recognized and understood, although the mechanisms of various emotions are different. However, it has the only certainty. American psychologist Paul Ekman proposed to divide emotions into six basic emotions: happiness, sadness, disgust, anger, fear, and surprise. This method of division is generally accepted and adopted by scholars. However, many researchers have found in the process of practice that the expression of individual emotions in the discrete emotion model is one-sided, cannot describe other emotions other than basic emotions, and has certain limitations. In addition, the discrete sentiment model cannot divide the intensity of human emotions at a certain moment.

2) Continuous sentiment model: Compared with the discrete sentiment model, the continuous sentiment model supports the continuous change of emotional state and accurately describes the emotion from spatial dimensions such as pleasure and arousal. In the two-dimensional continuous emotion model, the two dimensions of arousal and valence can best express the state of human emotion change. Therefore, the two-dimensional emotional ring model proposed by Russell is widely used in the research of emotional computing in recent years. The model uses arousal to express the strong to weak emotional state, and the valence is divided into positive and negative poles. Positive valence is used to express emotions in a pleasant state, while unpleasant negative emotions are expressed by negative valence. Figure 1 shows a schematic of the ring model.

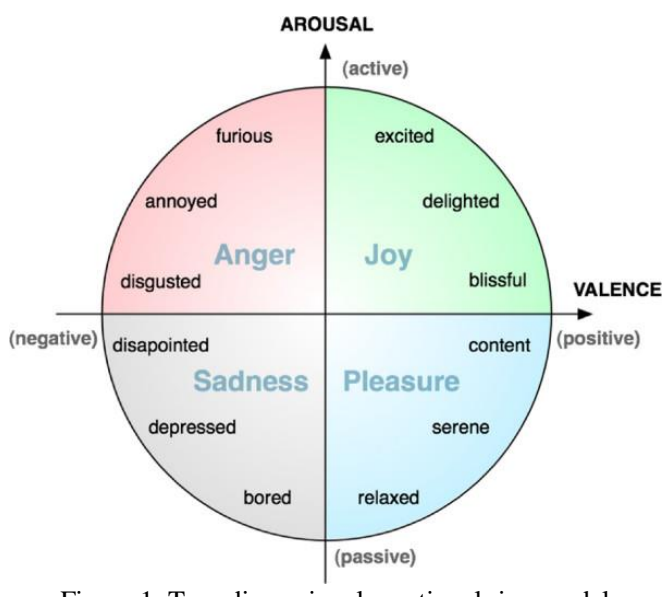

Figure 1. Two-dimensional emotional ring model

3) Cognitive-based sentiment model: The cognitive evaluation theory proposed by Dexi and Ryan in 1985 is currently widely used in the field of emotion recognition. Based on this theory, the OCC model is proposed in the book "Emotional Cognitive Structure", which defines 22 basic rules for the generation of basic emotions, and is currently the most representative cognitive evaluation emotion model. This model is different from the previous model of studying emotions from the perspective of psychology. It is based on cognitive structure. At present, the OCC cognitive evaluation emotion model is mainly used in the field of artificial intelligence emotion research.

In summary, discrete emotional models and continuous emotional models have their own strengths. The former is easy to understand and construct. The research process is not too complicated. The shortcoming is that the description of 
emotions is not delicate enough, and the classification is not detailed enough. In contrast, the latter is more relevant to human emotions in different environments and events. Therefore, this experiment applied a continuous emotional model and selected three kinds of calm, sad, and happy emotions as research objects.

Feature extraction is a very important part in the process of EEG signal recognition. At present, the feature analysis methods for EEG emotion recognition are mainly time domain analysis, frequency domain analysis, and time-frequency analysis. In recent years, people have gradually proposed wavelet analysis, convolutional neural networks, chaos analysis, and other methods.

1) Time domain analysis method: This method is the earliest developed EEG processing method to extract signals directly from the time domain. Time domain analysis focuses on analyzing the waveform, amplitude, and time history of EEG. Time domain analysis has the advantage of being intuitive and accurate.

2) Frequency domain analysis method: This method mainly analyzes the power spectrum of the EEG signal and converts the time domain EEG signal whose amplitude changes with time into the spectrum of EEG power with frequency. The electroencephalogram can visually reflect the distribution and changes of different frequency bands of EEG signals. Because of the non-stationary nature of EEG signals, it is generally necessary to segment first before analysis, and the AR model in the parametric model is simple in principle and small in computation, and it is suitable for real-time processing of short data.

3) Time-frequency analysis: The time window method or frequency window method is usually used in engineering technology to reflect the change of the frequency domain characteristics of EEG signals with time. As a powerful tool for analyzing time-varying non-stationary signals, it has become a hot spot in modern signal processing research. As an emerging signal processing method, it has received more and more attention in recent years. Commonly used methods include short-time Fourier transforms and wavelet transforms.

4) Nonlinear analysis method: At present, the nonlinear analysis of EEG signals is mainly based on the calculation of nonlinear dynamic parameters such as fractal value, complexity, approximate entropy, and bispectrum index, as well as the extraction of EEG characteristics.

5) Multi-dimensional statistical analysis method: This method can simultaneously process multiple weak EEG signals, which is beneficial to reveal and enhance the hidden features of these signals, and it has unique effects in weak EEG denoising and feature extraction. Commonly used methods are principal component analysis, factor analysis, and independent component analysis.

In summary, this experiment combines the principal component analysis method with the fractal dimension method to achieve higher stability and lower computational complexity than other calculation methods.

The current model methods for emotional EEG signals include artificial neural networks, decision trees, $\mathrm{K}$ nearest neighbors, and SVM.

1) BP artificial neural network: The BP neural network, which was proposed by Rumelhart et al., is a multilayer feedforward network capable of storing a large number of input-output mapping relationships. In this network, the calculation formula for expressing the mapping relationship does not need to be given in advance. The BP neural network has self-learning ability and fault tolerance within a certain range. Its learning algorithm consists of two forward and reverse propagation paths. If the input signal is forwardly propagated to the output layer and the error signal is within the fault tolerance range, then the learning ends. Otherwise, the error signal is propagated backward according to the original connection, and the parameters of each layer network are adjusted for reverse calculation, so that the error signal is reduced to the expected value. A large number of studies have proven that BP neural networks perform well in pattern classification.

2) Decision tree: Decision tree is a kind of easy-to-identify graphical analysis method that uses reference probability in decision-making. Because its constituent nodes and branches form a graph similar to many branches of a tree, it is called a decision tree. In many machine learning algorithms, the decision tree is mostly used as prediction data. By constructing a decision tree to obtain the probability that the expected value of the net present value is greater than or equal to zero, the attributes of the sample are judged, provided that the probability of occurrence of each case is a known condition. The advantage of the decision tree algorithm is that it is easy to understand and operate, and it does not need to have too much background knowledge. It can also make good processing for large data in a short time, but when there are too many categories to be identified, the error rate increases. 
3) K nearest neighbor algorithm: The advantage of the $\mathrm{K}$ nearest neighbor algorithm is that it is easy to understand and implement, and compared with other classification algorithms, only a very small number of adjacent samples can be used to complete the classification discrimination. The disadvantage is that the method is not suitable when the set and the categories are large.

4) SVM algorithm: SVM is a linear classifier in the classification problem. Its classification method is to construct a classification plane or a classification function. Through this function, two types of samples are segmented at both ends of the plane. For the phenomenon of dimensionality disaster caused by the input high-dimensional data in the traditional classifier, the SVM algorithm utilizes the mapping function of the kernel function from low dimension to high dimension to effectively solve the problem. The SVM algorithm solves the linear indivisibility problem in low-dimensional space due to the increase in dimension by introducing the kernel function. It also solves the linear indivisible problem in low-dimensional space by using the inner product of the kernel function. The optimal classification plane is constructed by a few support vectors that correspond one-to-one with the basis function, and some redundant samples are eliminated, thus avoiding the "dimensionality disaster" phenomenon that would occur in the previous classification process. SVM, with its unique advantages in solving nonlinear and high-dimensional samples, is the most widely used method in EEG signal pattern recognition.

In summary, through these classification methods, the SVM algorithm improves the generalization ability of the learning machine by minimizing the structural risk and solves the problem of over-fitting. The solution to the quadratic convex optimization problem is also the optimal solution. For the global category, the phenomenon of local optimal solutions in the pattern recognition process is avoided. The KNN is easy to understand and implement, and only a very small number of adjacent samples can be used to complete the classification. Therefore, this experiment applies the SVM algorithm and the KNN model and compares the output results.

\subsection{Experimental Design}

The EEG signal acquisition device used in the experiment is the acquisition platform of ESI Neu-roscan Company. It consists of four parts, which are used to play the stimulus material display, the head-mounted EEG collection cap, the signal amplifier, the data processing, and the host portion of the display. Considering that the effective signal duration is short and easily submerged under a large amount of useless data, a synchronous video camera is added to the original device to record the tester's face and limb movements so as to be as close as possible to the subsequent data. Effective data is grabbed, and the experimental platform structure is shown in Figure 2.

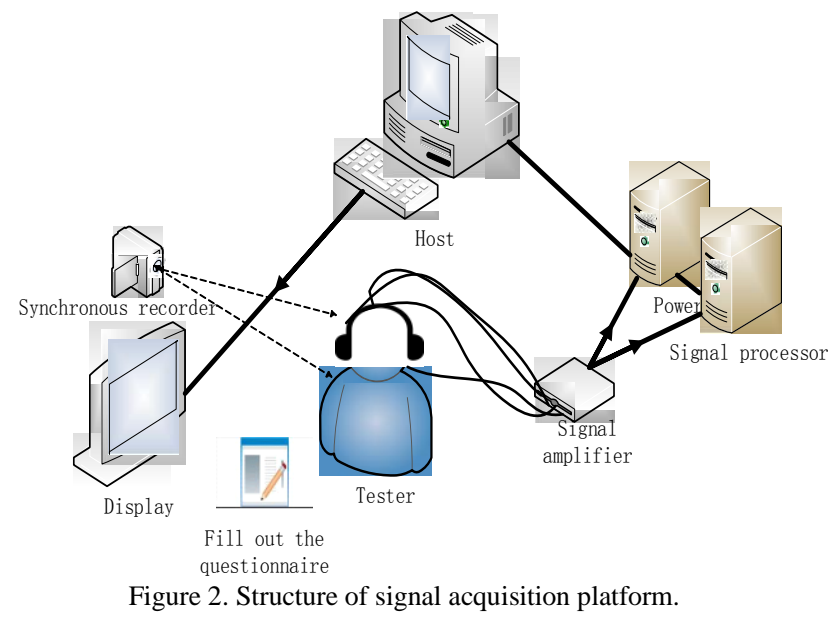

The purpose of the research is to find feature vectors that can distinguish different emotional states. The main content of the experimental design is to let the testers reach the states of joy, sadness, and calm under the stimulation of external film segments and record the electrical signals of their brains. The video is selected as the inducing material because the pictureinducing effect is not obvious and the individual differences are unstable, the music induces the tester's music literacy [6], and the video material can more effectively mobilize people's emotions. The selection of video materials in the experiment included the fragments of the sad material "Tangshan Earthquake" selected by the Shanghai Jiaotong Electric Database [7] and the popular video materials in Weibo. Since it takes a certain length of time to watch the video clip, the EEG signal that is really needed is easily buried in a large number of unwanted signals. Here, two links were specially designed. First, in the collection part of the experimental data, the synchronous video recording device was added to record the tester's facial 
expression. Secondly, the questionnaire was filled in. The design questionnaire contained questions about what kind of emotions were stimulated, the time period in which emotions appeared, and the degree of emotional realism, with the intention of allowing the tester to evaluate the validity of the data at the initial stage of the experiment and achieve initial screening of the data.

The experiment initially selected 30 college students aged 20 to 25 years old who were healthy and had no brain disease or brain surgery. Eight male students and four female students were selected as effective subjects. The experimenters wore a special 64-lead brain electricity collection cap, and the electrode placement position is shown in Figure 3. The experiment was started after the resistance of the electrode satisfied the requirements. The subject was required to sit on the head-up display in front of the computer. During the test, the tester was required to avoid large head and face movements as much as possible, to reduce interference of the EMG signal to the experimental data. Before each collection was a two minute period to allow the subjects calm down, and then the formal test process began. An emotional test cycle was about five minutes. The first was the ten second reminder time, followed by four minutes to play the stimulation clip. The questionnaire was filled in for 15 seconds, followed by a final ten second break. The experimental process is shown in Figure 4.

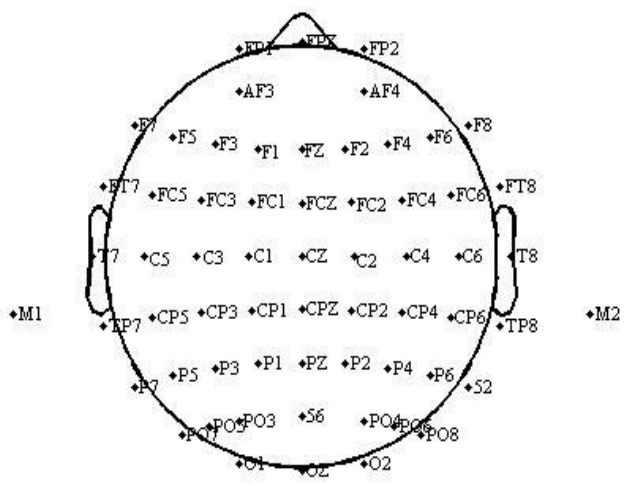

Figure 3. Electrode distribution map

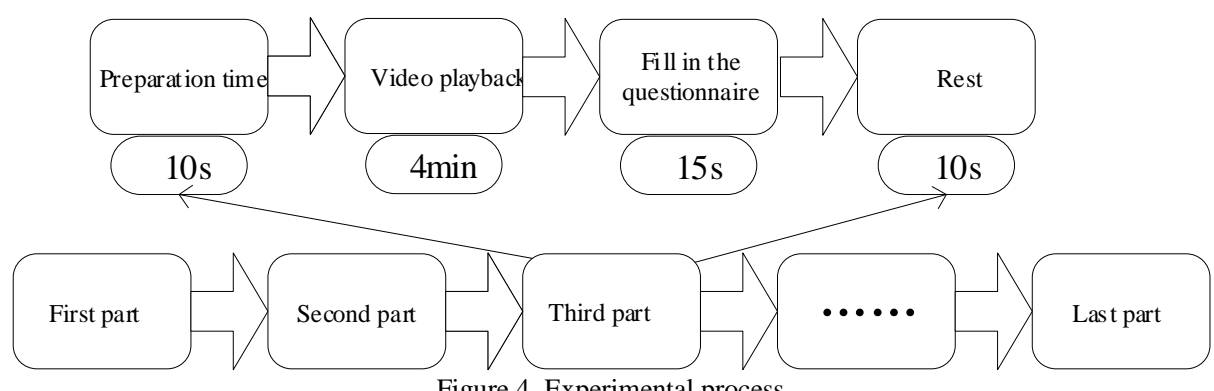

Figure 4. Experimental process

\section{Data Analysis}

The overall flow chart of this experiment is shown in Figure 5. Data analysis includes three major parts: preprocessing, feature extraction, and classification.

\subsection{Preprocessing}

Before data preprocessing, find useful data from the collected information, view the tester's questionnaire, watch the recorded video data from the original data, and intercept the valid data. First, determine whether the stimulus material is valid for the tester, and secondly, compare the video data. Capture valid data of the appropriate length and record it. Next, the data preprocessing part follows. We already know that the EEG signals related to emotions are mainly concentrated in the frequency band of $0-30 \mathrm{~Hz}$ [8], and the blink of an eye has a great influence on the EEG signals, especially the EEG signals of the forehead. Therefore, we must find and remove the blink signal from the useful signal. In the preprocessing stage of the EEG signal, our main task is to manually remove the obvious blink signal while filtering out the interference above $30 \mathrm{~Hz}$. At the same time, due to the original signal, the sampling frequency is $1000 \mathrm{~Hz}$. In order to reduce the amount of data calculation, we reduce the sampling frequency to $250 \mathrm{~Hz}$. Figures 6 (a) and (b) show the comparison between the original signal and the pre-processed EEG signal. After the pre-processing, the signal is more stable, and the interference is much less. 


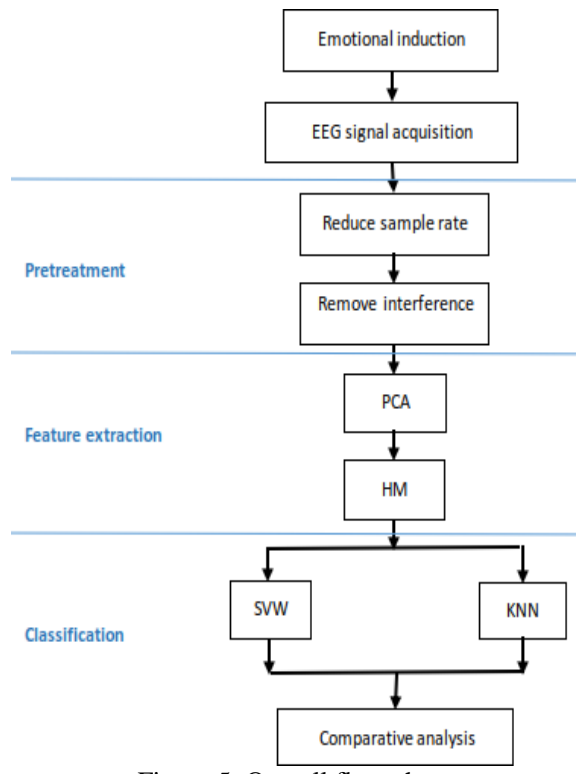

Figure 5. Overall flow chart

\subsection{Feature Extraction}

Principal component analysis is a multivariate statistical analysis. Construct a linear combination of the original variables to form new variables, so that the new variables can reflect the information of the original variables as much as possible irrespective of each other [9]. The variance is used to reflect the information carried by the data. The larger the variance, the more information that is carried. Firstly, the original data is normalized to eliminate the influence of the original variables due to dimensional differences and large differences between values [10]. Secondly, the correlation matrix is constructed to calculate the eigenvalues and eigenvectors. Finally, the cumulative variance contribution rate determines the number of principal components and generally takes the previous $p$ main component when the contribution rate is $75 \%-95 \%$.

The preprocessed data here is 64-dimensional. Even if the sampling frequency is reduced from $1000 \mathrm{~Hz}$ to $250 \mathrm{~Hz}$, the 64-channel signal contains a large amount of useless information. It is harmful and unfavorable for emotion recognition, and it will waste various resources. We use principal component analysis to reduce the dimension of the 64-lead signal to remove the less valuable information. Here, we also involve the problem of the cumulative contribution rate. In order to retain the useful signal as much as possible, the retention makes the cumulative contribution rate equal to or greater than the six principal components at $95 \%$. Figure 6(c) shows the partial signals that are arranged according to the eigenvalues from large to small after eigenvector transformation. It can be clearly seen that the variance of the corresponding components from larger eigenvalues is obviously larger than the other. The larger the variance gets, the more useful information there is in the signal.

Fractal dimension (FD) is a method to measure the complexity of data. The larger the fractal dimension, the more complex the signal. Conversely, the smaller the value, the more regular the signal [9]. The relevant dimensions are usually evaluated in the phase space for evaluation. Higuchi proposed an algorithm for estimating FD directly in the time domain without the need to reconstruct singular attractors [11]. HM also gives a reasonable estimate of the FD values for a shortterm time series. The HM algorithm is based on a known finite long time series $y=\{y(1), y(2), \cdots, y(N)\}$. It is a new time series, and $y_{m}^{k}$ is constructed as

$$
y_{m}^{k}=\left\{y(m), y(m+1), y(m+2), \cdots, y\left(m+\frac{N-m}{k}\right) \cdot k\right\}, \text { for } m=1,2, \cdots, k
$$

Here, $m$ and $k$ are integers representing the start time and time interval, respectively, and the length of each curve is calculated as

$$
L_{m}(k)=\frac{1}{k}\left\{\sum_{i=1}^{\left[\frac{N-m}{m}\right]}|y(m+i k)-y(m+(i-1) k)| \cdot \frac{N-1}{\left[\frac{N-m}{m}\right] \cdot k}\right\}
$$




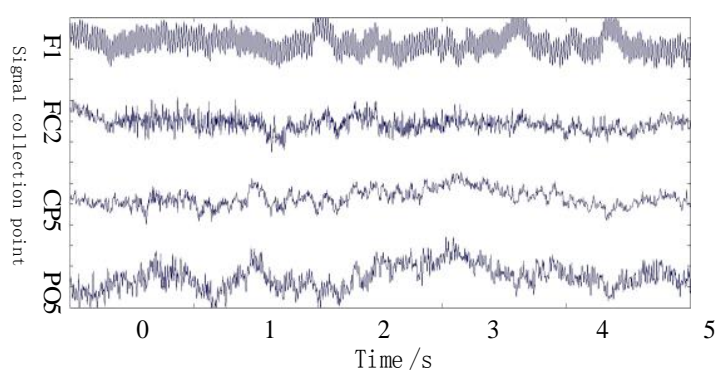

(a) Original signal

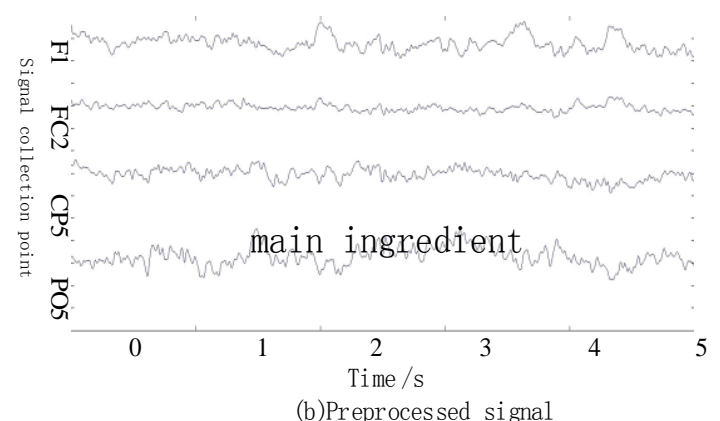

(b)Preproces sed signal

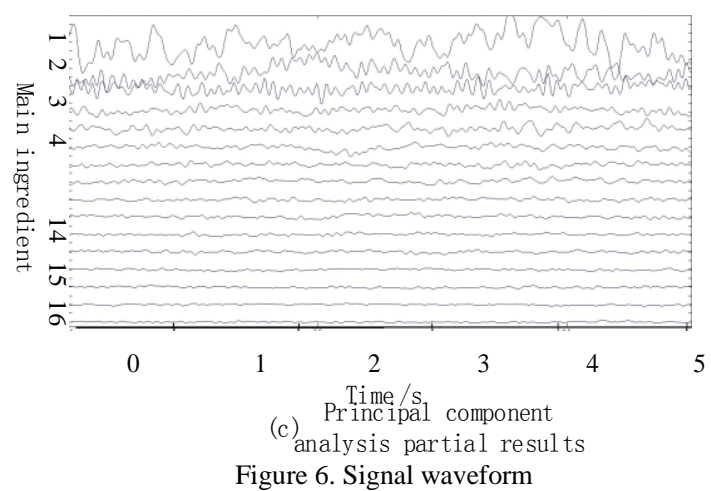

The interval $k$ and the length of the curve $L_{m}(k)$ are the means of the calculated curve. The relationship of this algorithm is $L_{m}(k) \propto k^{-D_{H M}}$, so we use the least mean square to fit $\log _{2} L(k)$ and $\log _{2} k$, calculate the negative slope value of the curve, and finally give the estimated value of FD. When calculating the value of the fractal dimension, the value of $k$ is 4 , the fractal dimension value of the 12 experimenters is calculated, and the broken line diagram is drawn. The simulation result is shown in Figure 7.

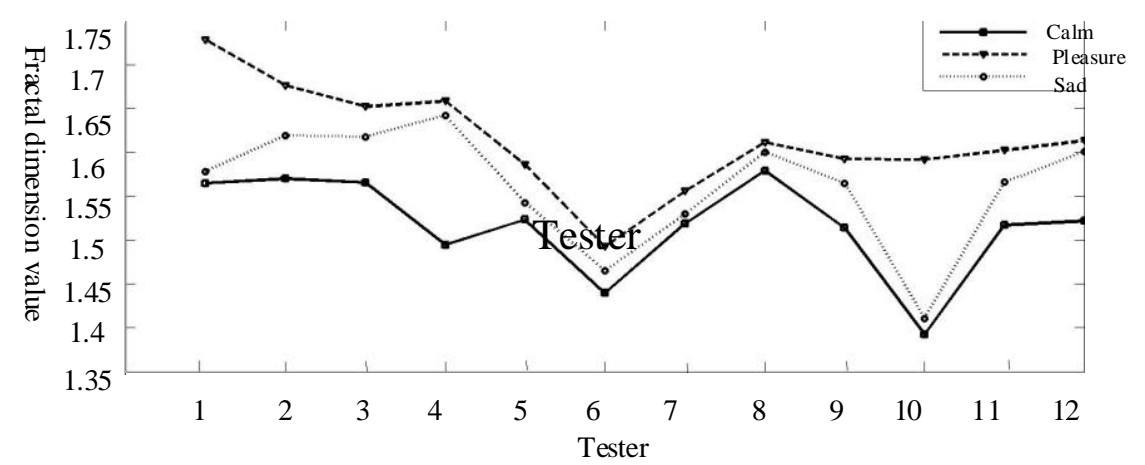

Figure 7. Fractal dimension distribution of three emotions of 12 testers

\subsection{Model Identification}

Support vector machine (SVM) is a data mining algorithm based on statistical learning theory. It has a wide range of 
applications in pattern recognition (distinguishing analysis, classification) and processing regression (time series analysis). SVM finds a hyper-plane that satisfies both the classification requirement and the optimal classification, so that the hyperplane obtains higher classification accuracy, and the white space on both sides of the hyper-plane is maximized [11].

KNN classification algorithm: First, calculate the distance between the training samples of the known category and the samples to be classified, find the $k$ nearest neighbors from the sample data points to be classified, and finally judge the categories of unclassified samples by the categories of these neighbors [10]. When the neighbors of the $k$ samples to be classified belong to a certain category, the sample to be classified is deemed to belong to the class. Otherwise, the categories of the $k$ sample are determined by the category with the largest number of neighbors. The distance of the sample in the KNN algorithm is derived from the Euclidean distance. The distance formula is

$$
d(X, Y)=\sqrt{\sum_{i=1}^{l}\left(x_{i}-y\right)^{2}}
$$

Where $X=\left(x_{1}, x_{2}, \cdots, x_{n}\right)$ and $Y=\left(y_{1}, y_{2}, \cdots, y_{n}\right)$ are samples. $N$ represents $n$ sample feature attributes. In the traditional algorithm, all the feature attributes of the sample are used to determine the distance between neighbors in the KNN algorithm, the dimensional disaster can easily occur [12], and the validity and accuracy of the classification are also affected. We choose support vector machine and the $K$-nearest neighbor classification algorithm to classify the data separately. The support vector machine algorithm is implemented by the LibSVM software package. It was developed by Lin et al. [13]. The KNN algorithm is based on Matlab. The three emotions were classified to obtain the classification accuracy, as shown in Table 1. The results show that SVM can make a more accurate judgment of happiness, while the KNN classification algorithm can make a more accurate judgment of calmness.

In order to test whether this experiment has a lower computational load, we performed the following comparative experiments. After the same pre-processing of the same EEG data, the wavelet packet transform was used to extract features from the pre-processed EEG signals to form a two-dimensional structure sample for training and testing by the classifier. Then, the convolutional neural network was used. The depth was the main line, and six CNN models were established from shallow and deep as emotion recognition classifiers to recognize emotions. The results obtained after averaging multiple measurements are shown in Table 2. The experimental results show that under the condition of similar precision, the EEG emotion recognition experiment based on fractal dimension can greatly reduce the time required, which greatly impacts the emotional classification system with high real-time requirements. contribution.

Table 1. Classification accuracy

\begin{tabular}{cccc}
\multicolumn{5}{c}{ Table 1. Classification accuracy } \\
\hline Emotion & Happy & Calm & Sad \\
\hline SVM Accuracy & $83.33 \%$ & $41.67 \%$ & $33.33 \%$ \\
KNN Accuracy & $75 \%$ & $83.33 \%$ & $58 \%$ \\
\hline \multicolumn{6}{c}{ Table 2. Classification accuracy } \\
\hline \multicolumn{6}{c}{ Accuracy } & Categories & Time \\
\hline Method & $83.33 \%$ & 3 & $46.13 \mathrm{~s}$ \\
This experiment & $85.56 \%$ & 3 & $98.95 \mathrm{~s}$ \\
\hline
\end{tabular}

\section{Conclusions}

This paper proposes an EEG feature extraction method based on fractal dimension. The pre-processed data is first reduced by the principal component analysis method to reduce the useless EEG signals. The reduced data is then used to obtain the fractal dimension values using the HM algorithm. In addition, KNN and SVM are selected to classify the fractal dimension values, and the output results are analysed and compared to obtain higher accuracy. Among them, under the support vector basis classification algorithm, the optimal precision is $83.33 \%$ of the happy state, and the optimal accuracy rate of the $\mathrm{K}$ nearest neighbour classification algorithm is calm state $83.33 \%$. Compared with other experiments, it can be concluded that the emotional classification experiment based on fractal dimension has a lower computational amount, which can significantly shorten the time required for emotional classification and is more suitable for real-time sentiment analysis. In the future, we can identify this method by integrating the brain-computer interface, adding it to the medical rehabilitation system, and quickly and easily obtaining the patient's emotional state or emotional record, in order to better achieve better treatment effects. 


\section{References}

1. B. Reeves and C. Nass, "How People Treat Computers, Television, and New Media Like Real People and Places," Cambridge University Press, CSLI, New York, 1996

2. C. Doukas and I. Maglogiannis, "Intelligent Pervasive Healthcare Systems," Advanced Computational Intelligence Paradigms in Healthcare - 3, Vol. 107, pp. 95-115, 2008

3. C. Lisetti, F. Nasoz, C. Lerouge, O. Ozyer, and K. Alvarez, "Developing Multimodal Intelligent Affective Interfaces for TeleHome Health Care," International Journal of Human-Computer Studies, Vol. 59, No. 1-2, pp. 245-255, July 2003

4. M. Murugappan, M. Rizon, R. Nagarajan, and S. Yaacob, "EEG Feature Extraction for Classifying Emotions using FCM and FKM," International Journal of Computers and Communications, Vol. 1, No. 2, pp. 21-25, 2007

5. K. Takahashi, "Remarks on Emotion Recognition from Bio-Potential Signals," in Proceedings of IEEE International Conference on Industrial Technology, pp. 186-191, Hammamet, Tunisia, December 2004

6. Y. P. Lin, C. H. Wang, T. P. Jung, T. L. Wu, S. K. Jeng, J. R. Duann, et al., "EEG-based Emotion Recognition in Music Listening," IEEE Transactions on Biomedical Engineering, Vol. 57, No. 7, pp. 1798-1806, 2010

7. R. Nie, X. Wang, and B. Lv, “A Review of Emotion Recognition based on EEG," Chinese Journal of Biomedical Engineering, Vol. 31, No. 4, pp. 595-606, 2012

8. Q. Wang and O. Sourina, "Real-Time Mental Arithmetic Task Recognition from EEG Signals," IEEE Transactions on Neural Systems and Rehabilitation Engineering, Vol. 21, No. 2, pp. 225-32, March 2013

9. S. Zhou, M. Mao, and J. Su, "Wind Power Prediction based on Principal Component Analysis and Artificial Neural Network," Power Grid Technology, Vol. 9, pp. 128-132, 2011

10. B. Yang, Z. Han, Q. Wang, and L. He, "Fractal Dimension Combined with RLS-ICA for EEG Signal Denoising," Journal of Zhejiang University, Vol. 7, pp. 1234-1240, 2014

11. T. Higuchi, "Approach to an Irregular Time Series on the Basis of the Fractal Theory," Physical D, Vol. 31, pp. 277-283, 1988

12. X. Xu, B. Lv, J. Song, W. Shi, Y. Hu, and S. Yan, "An Analysis Research for Digitized Features of Epileptic EEG using SVM," Applied Mechanics and Materials, Vol. 240, pp. 1169-1172, 2012

13. Z. Wang and K. Wang, "Improvement of K Nearest Neighbor Algorithm based on Entropy Weight," Computer Engineering and Applications, Vol. 30, pp. 129-131, 2009 